\title{
Material Characterization at High Strain by Adapted Tensile Tests
}

\author{
W.C. Emmens • A.H. van den Boogaard
}

Received: 28 June 2011 / Accepted: 21 November 2011 / Published online: 13 December 2011

(C) The Author(s) 2011. This article is published with open access at Springerlink.com

\begin{abstract}
The strength of materials at high strain levels has been determined using the so-called Continuous-Bendingunder-Tension (CBT) test. This is a modified tensile test where the specimen is subjected to repetitive bending at the same time. This test enables to create high levels of uniform strain. A wide variety of materials has been tested this way. The strength of the material after CBT testing has been measured in different ways: by secondary tensile tests, by interrupted CBT tests, and directly from the fracture in the CBT test. All methods yield similar results: the strength is largely unaffected by the cyclic pre-deformation and mainly depends on the overall increase in length. Only for multiphase materials the strength shows a minor influence of CBT test conditions. The hardening follows the extrapolated hardening observed in a conventional tensile test, except for brass. This test method can potentially be used for measuring hardening curves at high strain levels.
\end{abstract}

Keywords Material characterization $\cdot \mathrm{CBT}$ test $\cdot$ Adapted tensile test $\cdot$ Work hardening $\cdot$ Large strain

\section{Introduction}

In sheet metal forming operations, frequently high levels of strain are obtained. To analyze or simulate these operations, the material behaviour at these high levels of strain has to be known. The best known material characterization test is the tensile test, but the uniform strain in a tensile test is limited, and often much lower than obtained in practical forming

W.C. Emmens $\cdot$ A.H.van den Boogaard $(\bowtie)$

University of Twente,

Enschede, the Netherlands

e-mail: a.h.vandenboogaard@utwente.nl operations. To obtain material characteristics at higher levels of strain other tests are used, like compression tests, or characteristics are obtained from the diffuse neck in a tensile test.

Another approach is to use a classical tensile test but to suppress the instability that limits a tensile test in some way. This idea was first proposed by Taraldsen [1]. In his paper he discusses this approach in detail, and presents an adapted tensile test where during the test a set of two rolls is continuously moving up and down the specimen. These rolls supply a modest contact stress at the line of contact and in this way uniform elongations of up to $600 \%$ have been obtained. He actually used square and octagonal shaped specimens applying a complex set of rollers, but Rijken has showed that the same principle can be used on strip as well with only a single set of rollers obtaining $100 \%$ elongation [2]. A similar test was proposed by Benedyk et al. who, however, applied a set of three rolls as in a three point bending test, making it experimentally simpler [3]. The material is subjected to cyclic bending-under-tension, hence the test was called Continuous-Bending-under-Tension (CBT) test; both tests have been compared by Taraldsen in a later paper [4]. Benedyk proposed his test as a means to obtain high levels of uniform elongation, but did not study the properties of the material after performing the CBT test. He later proposed this test as a formability test [5].

The authors have used the CBT test in their laboratory from 2007 onwards to study effects of repetitive bending in incremental sheet forming (ISF). In previous papers results have been presented that showed that high levels of uniform elongation can be obtained easily. Levels of $200 \%$ are obtained for many materials, and levels of over $400 \%$ have been obtained in a few cases $[6,7]$. Recently also Allwood and Shouler [8] have applied this test, but as far as known to the authors the CBT test has not been used elsewhere. 
The aim of the present paper is to propose the CBT test as a new test method for determining the hardening relation of sheet metals at high levels of uniform elongation. As such this paper will pay much attention to experimental procedures and present a background for institutes that are considering using this type of test. As the material is subjected to cyclic bending, the effect of cyclic straining on the final material properties is a second subject of investigation. Basic Description of the CBT Test presents a general introduction to the CBT test. The test method has been described in detail in a previous paper [7], including the underlying mechanics and the phenomenology, so only a limited description is presented here. Strain History and Material Failure discusses in detail the strain history and strain state, as this differs from that in a conventional test and is relevant for the final material hardening. Basic Experimental Procedures and Material Selection present the general experimental procedures and material selection. Strain Measurements and Stress Measurements explain in detail how stress and strain have been measured. The results are presented in Results and discussed in Discussion of Results and Final Discussion and Recommendations. Proposal speculates on a simplified use of the CBT test, and final conclusions are presented in Conclusions.

\section{Basic Description of the CBT Test}

The CBT test is in fact a conventional tensile test carried out on a large tensile test specimen, whilst at the same time a set of three rolls is continuously moving up and down this specimen; this is schematically presented in Fig. 1. The

Fig. 1 Schematic representation of the CBT test and definition of some parameters. The specimen is held fixed at the top and is pulled downwards in the tensile testing machine, the roll set moves up and down bending of the strip by the rolls is considered to be bending-under-tension, meaning that the level of bending is so severe that at the concave side of the specimen the fibres are actually in compression. Note that the actual bending radius is larger than the roll radius; it is determined by an equilibrium between pulling force and bending moment and therefore depends on experimental conditions.

The simultaneous bending of the material reduces the tensile force required for plastic deformation. This ensures that only material actually being bent will deform and the test is in fact an incremental forming operation. The simultaneous bending also stabilizes the deformation process so that high levels of uniform elongation can be obtained; this stabilizing effect is discussed in detail in [9]. This test differs essentially from the conventional tensile test in some ways. As only material actually being bent will deform, only material 'visited' by the rolls will elongate. This means that in fact the gauge length remains constant being just the stroke of the roll set up-down movement. This supplies a simple relation between the logarithmic strain (NOT the technical strain!) and the total elongation:

$\varepsilon=\int \frac{d l}{l}=\int \frac{d l}{l_{0}}=\frac{1}{l_{0}} \int d l=\frac{\Delta l}{l_{0}}$

where $\Delta l$ is the elongation of the sample, and $l_{0}$ the up-down stroke [7]. This relation can be verified simply, see section 6 . Consequently: the maximum strain in a single test is equal to $\varepsilon_{\max }=s_{c b} / s_{u d}$, where $s_{c d}$ is the cross-bar stroke, and $s_{u d}$ the up-down stroke. Another aspect is that the strain increment of the material at each passage of the roll set is equal to

$$
\Delta \varepsilon=\frac{v_{\mathrm{cb}}}{v_{\mathrm{ud}}}
$$

where $v_{\mathrm{ud}}$ is the speed of the up-down movement and $v_{\mathrm{cb}}$ is the cross bar speed [6]. This means that the mechanics of the test is only determined by the ratio between cross-bar speed and roll speed, not by the individual speeds. The underlying mechanics is further discussed in detail in [7]. Anothervery relevant- difference with the conventional tensile test is that the strain state varies over the thickness of the specimen; this is explained in the next section.

To provide a general impression of the CBT test, a set of representative force-displacement curves for mild steel are presented in Fig. 2. These curves have been obtained with different cross-bar speeds, keeping all other parameters constant. In general the curves have the same shape as force-displacement curves from conventional tensile tests, in detail however they can be characterised as a set of alternating higher and lower plateaus between force peaks, clearly noticeable for example in the $3.5 \mathrm{~mm} / \mathrm{s}$ curve. The force peaks originate from the reversal of the roll movement, where momentarily the sample is elongated without bending. The higher plateaus occur when the roll-set is moving 


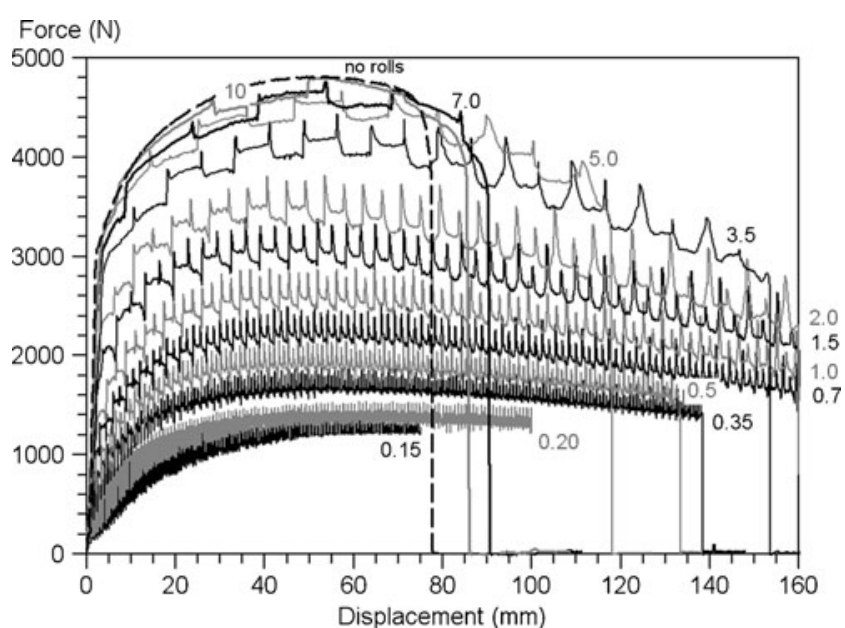

Fig. 2 A set of recorded force-displacement curves for mild steel, recorded at various cross-bar speeds. Legend: cross-bar speed in $\mathrm{mm} /$ s. The dashed line shows a curve obtained without rolls

away from the force transducer, the lower plateaus when the roll-set is moving towards the force transducer. In this way the individual up-down strokes of the roll set can be distinguished easily. The detailed shape of the force curves is discussed in [10]. At low cross-bar speeds the number of roll-set strokes to obtain a certain elongation is high, obviously, but also the force is clearly lower. This is a direct consequence of relation (2): lower cross-bar speeds require a lower strain increment per roll passage at the same amount of bending, for which a lower pulling force is required. This reduced force is caused by the fact that part of the crosssection is in compression and not by the Bauschinger effect (see next section). At very high cross-bar speeds the number of up-down strokes is just a few. At these speeds the test resembles a conventional tensile test and the recorded force is only slightly lower than when tested without rolls. A consequence of the high pulling force is that the elongation might not be longer restricted to the zones of bending, but that other parts of the specimen elongate as well. This creates a deviation from the relations (1) and (2) that where derived under the assumption that only material being bent will elongate.

\section{Strain History and Material Failure}

The CBT test is proposed to determine the hardening of sheet metal. In general the hardening depends on the strain history and the strain state of the material, and that is discussed in detail in this section.

As mentioned above the deformation process is bendingunder-tension (stretch bending) meaning that at the concave side the fibres are in compression. This means that the neutral line is located inside the strip. The situation is
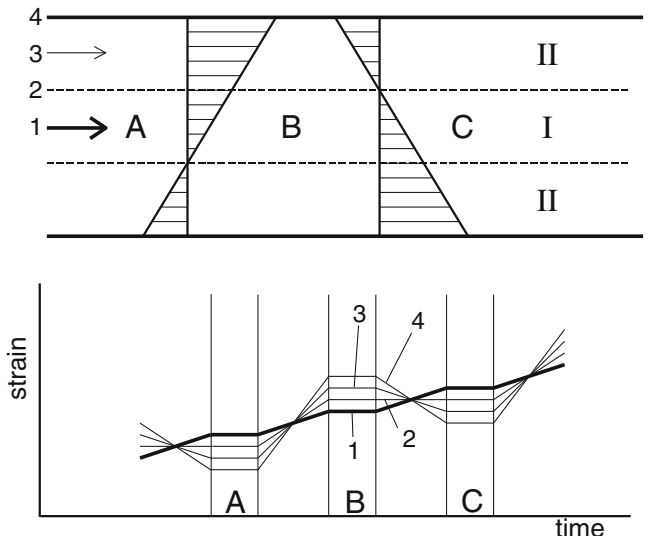

Fig. 3 Top: strain distribution over the thickness at a bending and consecutive reverse bending operation. Bottom: strain history for four points

visualized in Fig. 3. The top figure presents the strain distribution in a bending and consecutive reverse bending operation with simultaneous stretching, for example occurring in a situation were at $\mathrm{A}$ and $\mathrm{C}$ the strip is straight and at $\mathrm{B}$ the strip is curved. The figure shows that in the centre of the strip (zone I) the material is subjected to monotonous stretching only. However in the zones near the surface (labelled II) the material is subjected to repetitive tension and compression evoking strain reversal. The strain history for the locations 1-4 is presented in Fig. 3, bottom. In the outer zones II the material is subjected to repetitive reverse straining (ratcheting). This affects the stress level in zone II by the occurrence of the Bauschinger effect. The width of the central zone varies from case to case. In tests carried out at low pulling speeds it can be very small, estimated only $10 \%$ of the total thickness. In tests carried out at high speeds it can occupy almost the whole strip thickness.

The cyclic straining in the zones II can be further noticed in Fig. 4 that shows the stress history for three points during one complete up-down cycle of the roll set; one in the centre of the specimen and two near the surface (lower and upper), the data

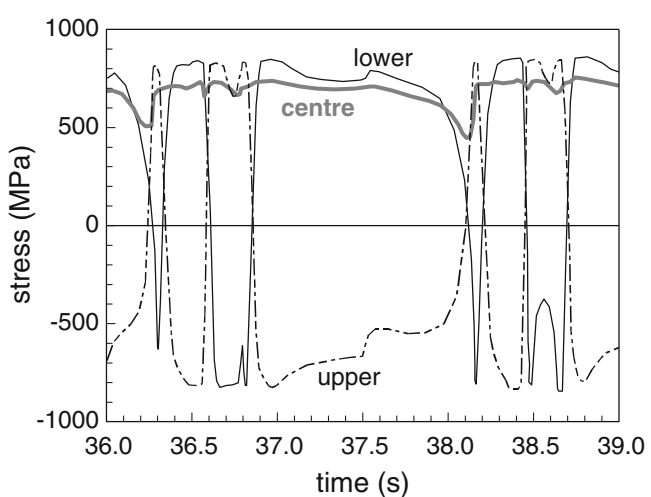

Fig. 4 Stress history for three points during a complete up-down cycle. Material: mild steel 
are obtained from FEM simulations described in detail in [10]. The two passages of the roll set can be easily distinguished, one at roughly $36.5 \mathrm{~s}$, the return at roughly $38.5 \mathrm{~s}$.

The graph shows that of the two points near the surface always one is in tension, the other is in compression. This indicates that at all times part of the cross-section is in compression but this situation changes eventually. During a test the relative width of zone I increases gradually as the strip thins constantly. If the test continues the neutral line for bending finally shifts out of the material, reducing the width of the zones II to zero, meaning that the whole cross-section is in tension. From that moment on the test continues as a conventional tensile test, albeit on highly deformed material, creating rapid fracture. This failing mechanism could also be observed in the FEM simulations from which Fig. 4 was taken, see [10] for more details. This type of failure is called failure by instability, it occurs when the stabilizing effect of the bending operations is lost. A typical characteristic is that for specimens failing by instability the elongation at fracture decreases with increasing cross-bar speed.

As the material is subjected to cyclic straining failure by low-cycle fatigue is possible as well. This indeed occurs with all materials at low testing speeds and is the reason that fatigue sensitive materials like aluminium and leaded brass show relatively poor overall performance in this type of test. A typical characteristic of this type of failure is that the elongation at fracture increases with increasing cross-bar speed.

\section{Basic Experimental Procedures}

The CBT test requires a tensile testing machine with an additional drive for the roll-set movement. The set-up that was used by the authors is shown in Fig. 5.

The machine is an MTS 810 hydraulic tensile testing machine. This allows high velocities and fast response in feed-back mode, but the stroke is limited by the hydraulic cylinder to max. $160 \mathrm{~mm}$. This also dictates the usable updown stroke, as that determines the length strain, see equation (1). A default stroke of $140 \mathrm{~mm}$ was chosen, this allows a maximum true strain of $160 / 140=1.14$ and creates a zone of uniform strain of approx. $10 \mathrm{~cm}$ length (see Fig. 8). This strain limit was sufficient for most materials, but notably for mild steel and stainless steel smaller up-down strokes had to be applied occasionally as these materials can be stretched to higher strains. Presumably a fast mechanical testing machine with larger stroke is better suited.

A compact and simple roll-set was constructed. This allows only variation in the roll penetration P (see Fig. 1). This setting determines the nominal bending angle $\alpha$ as in Fig. 1, this varied from $2.7^{\circ}$ to $18^{\circ}$ assuming a membrane situation. The roll set was attached to a computer controlled

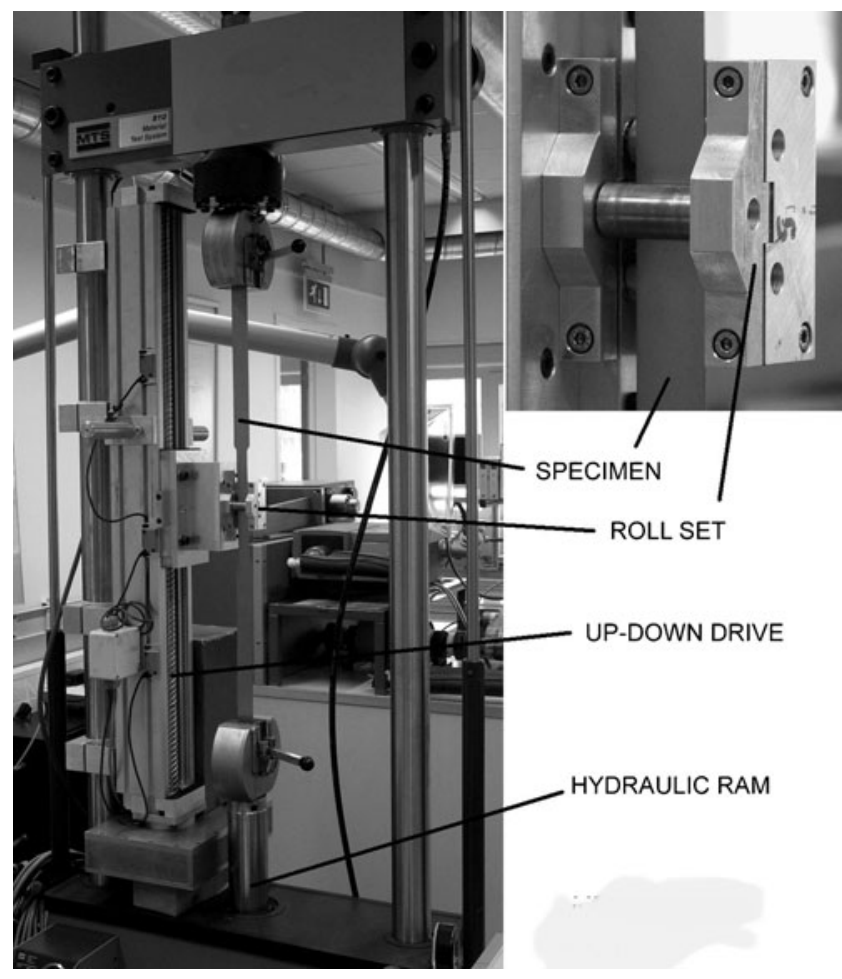

Fig. 5 Equipment used for the tests. See Table 1 and Fig. 6 for dimensions

INA linear drive using a high-precision screw shaft, similar to mechanical testing machines. This up-down drive was very large and was situated inside the frame. Therefore the jaws could not come close together. This has been solved simply by applying very large specimens of which only a small part is actually used. The device had a vertical load limitation of only $1,000 \mathrm{~N}$, which restricted testing of highstrength materials.

The dimensions of the specimen are shown in Fig. 6. The elongation of the wider parts could be ignored except for soft brass and stainless steel that show high levels of work hardening. These materials showed occasionally considerable elongation of the wider parts in high-speed tests. In this paper sample elongation always means: the elongation of the central, narrow part.

A test series comprises a series of tests with various cross-bar speeds; the up-down speed was held constant at $66 \mathrm{~mm} / \mathrm{s}$ in all tests. The cross-bar speed is limited at the upper side by the condition that at least one complete updown stroke should be performed, and at the lower side

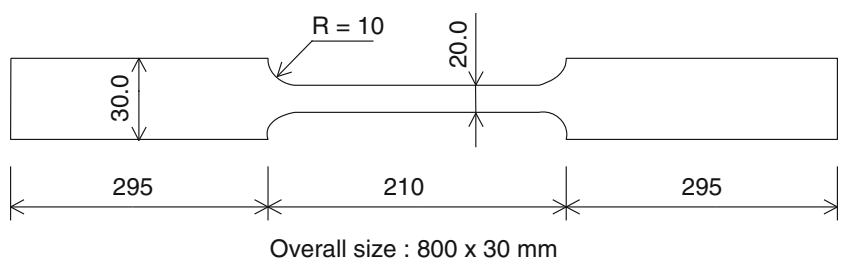

Fig. 6 Dimensions of the specimen, not to scale 
mainly by time considerations, the slowest test took roughly $15 \mathrm{~min}$. The number of up-down strokes in a single test varied from just a few, to roughly two hundred.

Most tests, including those shown in Fig. 2, were performed at constant cross-bar speed as in a conventional tensile test. The following procedure evolved after some preparatory tests: a sample is placed in the machine and aligned; a small tension load of $100 \mathrm{~N}$ is applied and kept constant while the roll-set is tightened; the roll-set is started, initially still under force control and one complete up-down stroke is performed, mainly to see if the clamping by the self-acting jaws is secure; after that the cross bar movement is started also and the test continues under constant speed control. Contrary to conventional tests, the CBT test can also be performed as a constant force test. The procedure is as follows: a sample is placed in the machine and aligned; a small tension load of $100 \mathrm{~N}$ is applied and kept constant while the roll-set is tightened; the final load (either below of above the yield stress) is applied and kept constant during the whole test under force feed-back, after a few seconds the roll-set is started as well and the sample elongates. This has only been done in a limited number of cases that are indicated specifically. This procedure can probably only be performed using hydraulic machines as the feed-back system must correct the force peaks shown in Fig. 2 by adjusting the cross-bar speed rapidly. Constant force tests are particularly suitable for tests at high speed, where the pulling force is only slightly lower than the material strength.

The test continues either until fracture or until a prescribed displacement is obtained. In a few cases the test was performed as a constant speed test, but after a certain elongation the roll-set was stopped while the test continues, from that moment on as a conventional tensile test causing rapid fracture. These tests are called interrupted test, and some examples of recorded force-displacement curves are shown in Fig. 7. In this figure A, B and C are interrupted tests; D is an uninterrupted test, tested until fracture. Preliminary tests showed that it makes no difference if the roll set is just stopped and remains tightened, or is removed completely.

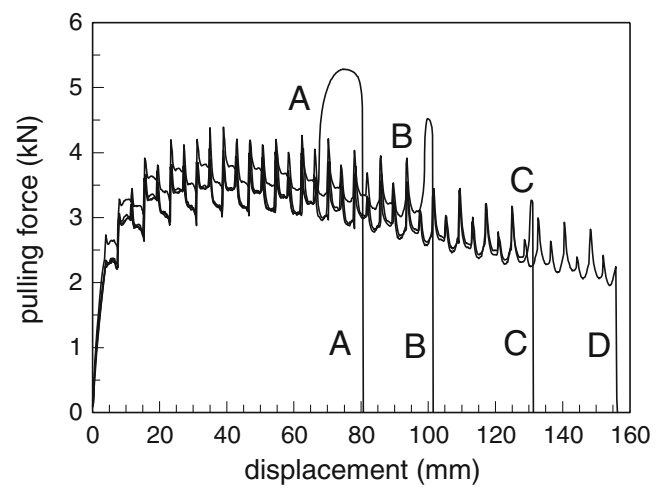

Fig. 7 Force-displacement curves of some interrupted tests (mild steel)
In the test, one end of the specimen is held stationary and the other end is attached to the moving 'cross-bar'. This creates an asymmetric shape as shown in Fig. 8. The deformation is concentrated into the zone that is repetitively bent by the moving rolls indicated by the rectangle in Fig. 8 of approx. $10 \mathrm{~cm}$ length. Below that (to the right in Fig. 8) a tapered section originates from material that is moved out of the deformation zone at increasing strain levels.

Finally, a complete overview of testing conditions is presented in Table 1.

\section{Material Selection}

A wide variety of materials has been used in the test allowing thorough conclusions about influence of material. The selection comprises bcc, fcc and hcp crystal structures, single phase and dual phase structures, materials that deform by twinning (brass, possibly titanium), and variations in thickness, formability and pre-deformation. The tests were carried out in close co-operation with Tata Steel, Netherlands, who supplied all the low-C steel and aluminium variants, and performed all the material characterization and the grid measurements. The stainless steel was obtained directly from the manufacturer Outokumpu, Finland, the brass and titanium materials were obtained on the commercial market.

An overview of all materials tested is presented in Table 2. All data have been obtained in standardized tensile tests (Euronorm), except for $m$. The strain-rate hardening coefficient $m$ is defined as $m=\operatorname{dlog}(\sigma) / \operatorname{dlog}(\dot{\varepsilon})$, this was determined from the force maxima (UTS) in three tensile tests carried out at $0.2,1$ and $5 \mathrm{~mm} / \mathrm{s}(v)$ respectively by fitting a straight line through the log (UTS) $\log (v)$ data. Material SS1 shows an anomalous strain-rate dependency: carrying out separate tests with different speeds yields another result than abruptly changing the speed in a single test. The force maxima showed a negative strain-rate hardening, but the force at lower strains showed a positive effect. This is probably due to the temperature dependent transformation from austenite to martensite. The amount of post-uniform elongation indicates that the momentary strain-rate hardening is positive.

\section{Strain Measurements}

The aim of this paper is to study material strength at high strain levels, so both stress and strain have to be determined. The previous sections have mentioned a zone of uniform deformation, see Fig. 8. The question is now how uniform the strain is in that zone. A thorough investigation would be to map the strain in that zone completely by using measuring 


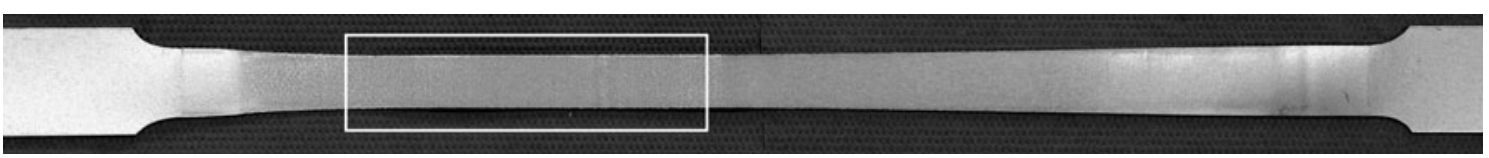

Fig. 8 Specimen after testing. The left-hand side was held stationary, the right-hand side was attached to the moving cross-bar. The rectangle indicates the zone of uniform elongation

grids, either regular or irregular, by well known techniques. However this is difficult for various reasons:

- The high level of strain exceeds the limits of some techniques

- The severe level of roughening of the surface makes detection difficult

- The frequent passing of the rolls might damage the grid

- The presence of the roll set hinders real-time optical measurements

A single attempt has been made by applying a regular grid of $2 \mathrm{~mm}$ spacing on a mild steel sample. The grid was etched into the surface being the most robust way to apply a grid, but only incomplete results could be obtained due to severe deterioration of the grid. The major strain at the centre of the strip in the narrow section (see Fig. 6) is presented in Fig. 9. The position is measured from the top; the orientation is similar to that in Fig. 8. This figure shows that in the tapered part, roughly at positions $170-320 \mathrm{~mm}$, the strain varies linearly with position. This is an indirect confirmation of equation (1).

The variation of strain over the width of the specimen in the tapered part is presented in Fig. 10. From this figure it was concluded that within the accuracy of the measurements the strain is uniform over the width of the specimen.

The mean strain can also be determined by measuring both the width and thickness of the specimen after the test. This has been done by hand for all samples. The width was measured using a vernier calliper of $0.05 \mathrm{~mm}$ resolution and turned out to be constant over the uniform zone within $0.1 \mathrm{~mm}$. This means that the error in width measurement is less than $1 \%$ which is acceptable. The thickness was

Table 1 Overview of all experimental conditions

\begin{tabular}{ll}
\hline Material & see Table 2 \\
\hline Specimen size & see Fig. 6 \\
Up-down speed roll set & $66.7 \mathrm{~mm} / \mathrm{s}$ (constant) \\
Up-down stroke roll set & $140 \mathrm{~mm}$ (default), 120, 110, 100, 80 mm \\
Roll diameter D (Fig. 1) & $15 \mathrm{~mm}$ \\
Roll distance L (Fig. 1) & $35 \mathrm{~mm}$ \\
Roll penetration P (Fig. 1) & $2.3 \mathrm{~mm}$ (default) 0.3, 1.3, 3.3, $4.3 \mathrm{~mm}^{*}$ \\
Pulling speed & $0.10 \mathrm{~mm} / \mathrm{s}-20 \mathrm{~mm} / \mathrm{s}$ \\
Max elongation & $160 \mathrm{~mm}$ (limited by machine) \\
\hline
\end{tabular}

*Roll penetration P differs for material MS1 measured with a conventional screw thread micrometer of $0.005 \mathrm{~mm}$ resolution. This was more difficult to perform due to non-flatness of the specimen which is caused by the facts that after the tests the specimen is curved, and in the final stages shows transverse bands, see [7]. Careful measuring showed that the thickness is uniform within $0.02 \mathrm{~mm}$, for many specimens even $0.01 \mathrm{~mm}$. This means that the inaccuracy in thickness measurement is generally in the range 1$5 \%$. A representative collection of resulting width strain and thickness strain for material MS1 is shown in Fig. 11; this presents a good impression of the scatter. Note that the ratio between width strain and thickness strain varies with experimental conditions, notably with the centre roll penetration $\mathrm{P}$ (see below). However, it is likely that also a systematic error is created, as due to the roughening and non-flatness the thickness might be measured a little too high. Note also that a non-uniform thickness (and consequently: strain) may arise at the final stage of the test prior to failure, due to multiple necking, see [7].

From the width strain and thickness strain the length strain was determined assuming constant volume, and subsequently the von Mises effective strain. The assumptions underlying this test predict that the length strain is proportional to the sample elongation according to equation (1). In Fig. 12 the results for material MS1 are presented, where however the elongation has been converted to an up-down stroke of $140 \mathrm{~mm}$ when a lower up-down stroke has been applied in the test. Example: if the specimens failed at $150 \mathrm{~mm}$ elongation with an up-down stroke of $120 \mathrm{~mm}$, the converted elongation for a $140 \mathrm{~mm}$ stroke is equal to $150 \times 140 / 120=175 \mathrm{~mm}$.

The dashed line in Fig. 12 shows the theoretical relation (1) and the measured strains agree satisfactorily with that. This confirms the assumption underlying this test that only material being bent will actually deform, unless the pulling force is so high that other parts in the narrow section deform as well (these cases are not shown in the figure). From these results the authors have concluded that determining the length strain by width and thickness measurements is sufficiently accurate for the purpose of the experiments, corroborated by the results shown in section 8 , and that indeed the deformation is sufficiently uniform.

The results presented in Fig. 11 show that the width strain is lower than the thickness strain (in absolute values), indicating that the strain state shifts towards plane-strain in the test. The strain state is characterized by the ratio between 
Table 2 Overview of materials tested

\begin{tabular}{|c|c|c|c|c|c|c|c|c|c|}
\hline $\mathrm{Nr}$ & Description & Code & thickn. (mm) & YS (MPa) & UTS (MPa) & unif. elong (\%) & $\mathrm{r}$ & $\mathrm{n}$ & $\mathrm{m}$ \\
\hline MS1 & mild steel DC04 & & 0.80 & 161 & 288 & 22 & 2.54 & 0.21 & 0.017 \\
\hline MS2 & mild steel DC04 & & 0.97 & 159 & 296 & 24 & 2.15 & 0.22 & 0.016 \\
\hline MS3 & mild steel DC06 & & 0.70 & 143 & 301 & 23 & 2.51 & 0.23 & - \\
\hline MS4 & mild steel DC04 & & 0.70 & 164 & 305 & 22 & 2.22 & 0.21 & 0.014 \\
\hline MS5 & mild steel DC04 & & 0.96 & 185 & 309 & 20 & 2.51 & 0.19 & 0.016 \\
\hline MS6 & mild steel DC04 & & 0.76 & 340 & 354 & 6 & - & 0.10 & - \\
\hline DP1 & DP steel; DP600 & & 0.99 & 387 & 667 & 14 & 0.98 & 0.15 & 0.006 \\
\hline $\mathrm{SS} 1$ & stainl. steel SS304 & 1.4301 & 0.80 & 320 & 695 & 62 & 0.98 & 0.51 & \pm 0.011 \\
\hline AL1 & alum. AA5182 & & 1.15 & 124 & 287 & 22 & 0.66 & 0.32 & -0.009 \\
\hline AL2 & alum. AA6016-T4 & & 0.96 & 109 & 233 & 18 & 0.64 & 0.25 & 0.005 \\
\hline AL3 & alum. AA6016-T4 & & 1.10 & 145 & 261 & 22 & 0.70 & 0.25 & -0.001 \\
\hline BR1 & brass $63 / 37$, soft & 2.0321 & 0.69 & 130 & 330 & 51 & 0.69 & 0.55 & 0.000 \\
\hline $\mathrm{BR} 2$ & brass $63 / 37$, medium & 2.0321 & 0.70 & 250 & 500 & 35 & 0.70 & 0.36 & -0.002 \\
\hline BR3 & brass $58 / 39 / 3$, hard & 2.0401 & 0.69 & 520 & 610 & 8 & 0.71 & 0.08 & -0.002 \\
\hline TI1 & titanium grade 2 & 3.7035 & 0.78 & 400 & 439 & 10 & 4.0 & 0.15 & 0.023 \\
\hline
\end{tabular}

1: material MS6 is material MS1 pre-strained to $\varepsilon_{\mathrm{x}}=0.18 .2$ : material DP1 is zinc-coated, all other materials are uncoated. 3: material BR3 is leaded brass, materials BR1 and BR2 ordinary yellow brass

width strain and thickness strain, just as for the $r$-value (Lankford's parameter) in the conventional tensile test. The measured value depends on the centre roll penetration, and can both decrease or increase with increasing speed. The mean values for each depth setting have been determined and these are presented in Fig. 13 as an 'apparent r-value'. The values for the as-received material are presented as negative settings, the setting to ensure that there is just no bending (penetration= - thickness). The graph shows that all materials more or less show the same behaviour although mild steel seems more affected than the other materials. There is a tendency to shift towards plane strain $(r=0)$, and more for a deeper penetration (read: larger bending angle). It should be emphasized that the strain state varies over the width of the specimen, the values presented in Fig. 13 only being an average. At the very edges the strain state is always uniaxial. This different state at the edges could be seen by close examination of the specimens, see [7].

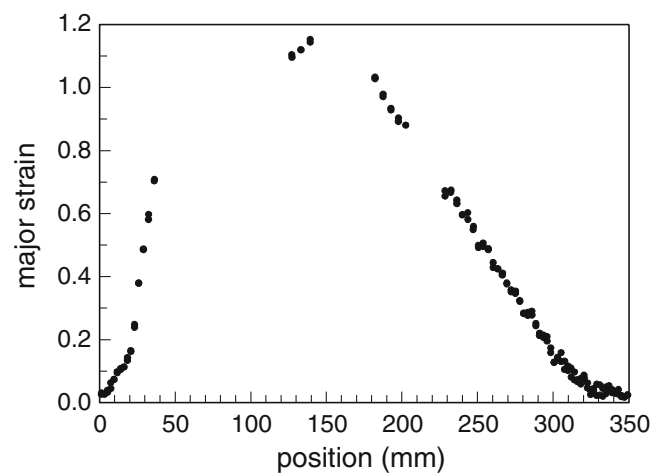

Fig. 9 Major strain in the centre of the specimen

\section{Stress Measurements}

The strength after testing (level of hardening) has been determined in three ways, described in the following sections. The strength has been obtained both from specimens pulled until fracture, and from specimens where the CBT test was simply stopped after reaching a certain elongation.

\section{Secondary Tensile Test}

The most rigorous way to determine the strength after the CBT test is to perform an additional, conventional tensile test. This has been done by cutting a small sample out of the tested specimens as shown in Fig. 14. The geometry was chosen simply as it was a Tata Steel in-house standard size and fit for

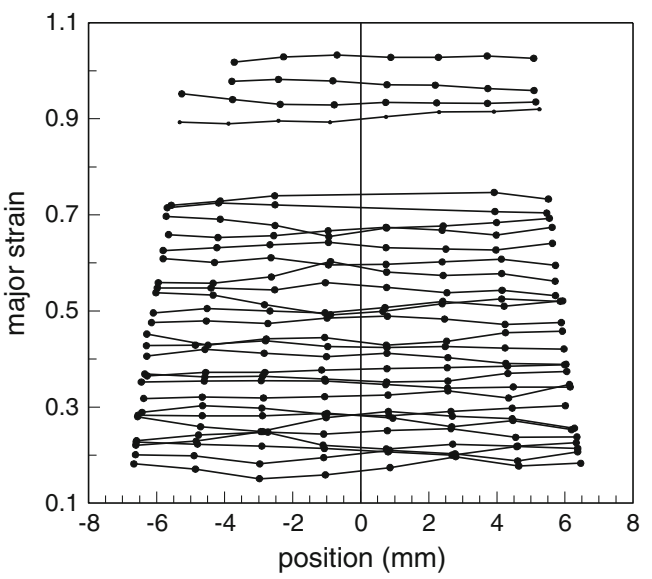

Fig. 10 Variation of major strain over the width of the specimen 


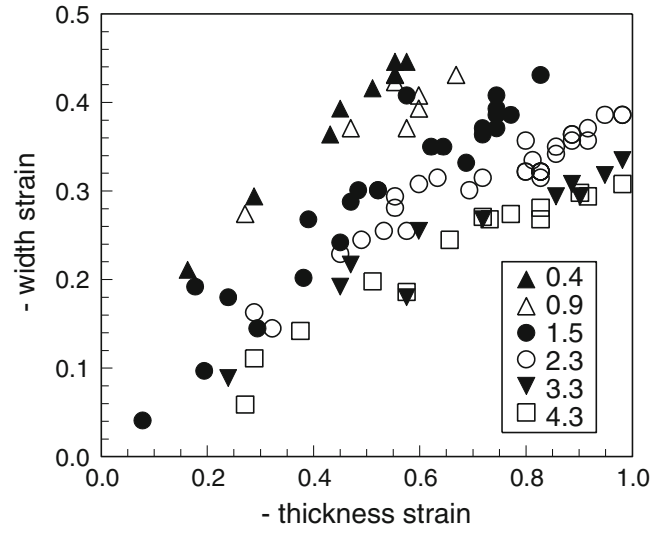

Fig. 11 Width strain and thickness strain for material MS1. Symbol type denotes centre roll penetration in $\mathrm{mm}$

the purpose. These tests can only be performed on specimens where the uniform part is without visible defects like small necks, and for fractured specimens where the fracture leaves a sufficiently long uniform part. In several occasions the specimens showed premature failure in the secondary test, meaning that the force-displacement curve did not show a proper maximum as shown in Fig. 15, left. This is presumably caused by damage that was already accumulated during the CBT test, and occurred notably with aluminium; these results have been discarded. Only results from tests showing a proper force maximum as shown in Fig. 15, right, have been used, and from these tests the UTS and uniform elongation were used to determine the overall strength. This method presents the most accurate results, but requires also the most effort.

These secondary tests have also been performed on asreceived material, and on samples pre-strained without bending, for comparison.

\section{Specimens Failed by Instability}

If the test is not interrupted and does not fail by fatigue, it finally ends by instability as explained in Strain History and

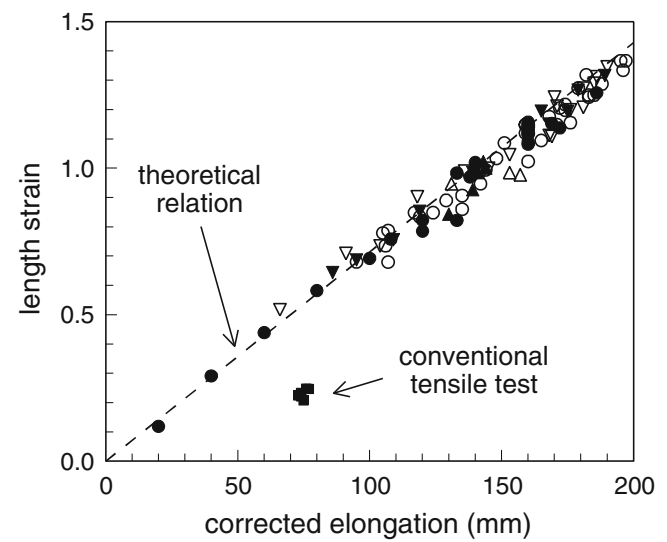

Fig. 12 Length strain plotted as a function of elongation for material MS1. The symbol type denotes centre roll penetration as in Fig. 11

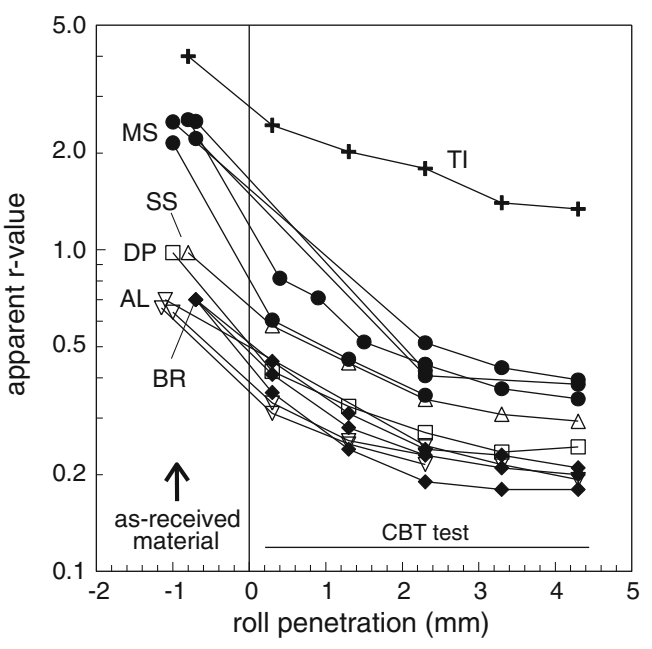

Fig. 13 Strain state in the specimens measured after the test. Symbols denote material class. Negative settings refer to the undeformed material

Material Failure. In that case, in the very final stage of the test the whole cross-section of the specimen is subjected to tension and not only the centre part. This means that from that moment on the mean tension stress (force / area) is equal to the mean flow stress of the material, averaged over the whole cross-section. In general the sample will fail quickly as can be seen for example in the FEM simulations presented in [10]. The stress and strain at fracture have been determined for all samples for which it was clear that indeed failure was by instability, but this occurred not to the same extent for all materials. A necessary condition is that the fracture is at an oblique angle as in a conventional test (see [7] for a discussion on fracture types). The cross-section area has been determined by measuring width and thickness of the uniform part as described in Strain Measurements. If the sample showed a clear diffuse neck (notably for titanium) the cross-section of that neck was determined in the same way and used instead. The force at fracture was determined from the measured force-displacement curves, but this was not always simple. In many cases the tests ends on a force peak and the force at fracture is simple to determine; see for example curve D in Fig. 7, and also the $3.5 \mathrm{~mm} / \mathrm{s}$ curve in Fig. 2. In some cases the force decreases gradually at fracture

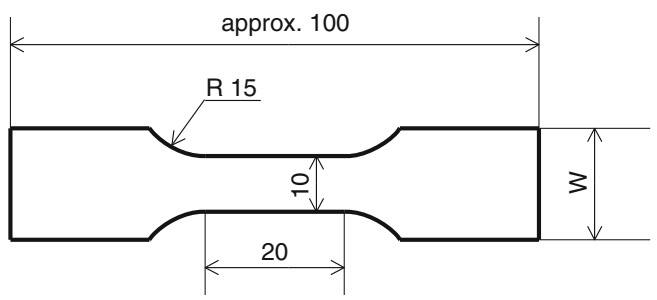

Fig. 14 Dimensions of samples for the secondary tensile tests. The width $\mathrm{W}$ is the width of the samples from the CBT tests. The narrow part is machined 

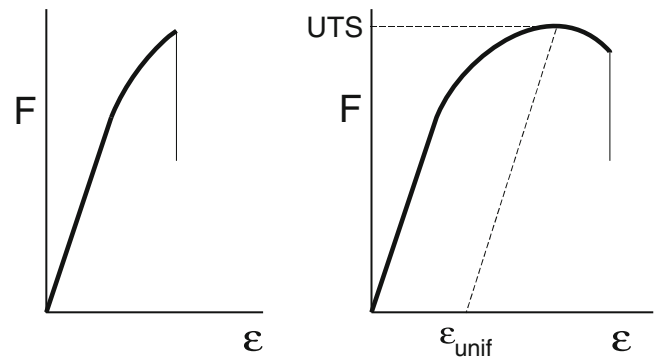

Fig. 15 Results from secondary tensile tests, schematically. Left: sample showing premature failure, right: sample showing a proper force maximum

as for example shown in the $5 \mathrm{~mm} / \mathrm{s}$ and $7 \mathrm{~mm} / \mathrm{s}$ curves in Fig. 2. In those cases it is not clear what the 'force at fracture' is exactly, and this creates an additional inaccuracy. From these data both stress and strain at fracture are determined, but the accuracy is lower than with the secondary tensile tests.

\section{Interrupted Tests}

Another way is to perform interrupted tests that have already been mentioned in Strain History and Material Failure. Examples have been presented in Fig. 7 showing actually recorded force-displacement curves. Curves A, B and C are interrupted tests; note the difference in elongation until fracture after interruption. Curve D is from an uninterrupted test.

The interrupted test can be regarded as an in-situ secondary test. The strength is determined from the pulling force at fracture, and the cross-section area of the specimen is determined as described in section 7.2. This method has been applied on only a few samples of two materials.

\section{Results}

The results for material MS1 are presented in Fig. 16. The figure presents both stress and strain. The stress is

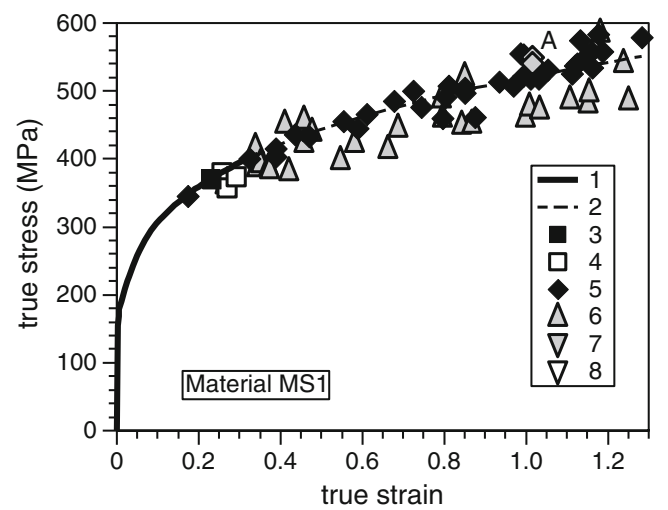

Fig. 16 Constructed true stress - true strain curve for material MS1. Symbol type denotes item type as described in the text determined as described in the previous section. The strain is always the von Mises effective strain based on the increase in length of the specimen, and NOT the cumulative incremental strain. For results obtained from secondary tensile tests it is the effective strain of the specimen after the CBT test, plus the uniform strain measured in the secondary test. Unless mentioned otherwise, all tests are constant speed tests.

The following items can be found in the following graphs:

1. a stress-strain curve derived from a secondary test on as-received material;

2. an extrapolated stress-strain curve assuming power-law hardening using $C$ and $n$ from the measured curve, only for low-C steel;

3. points derived from the UTS in secondary tests on asreceived material;

4. points derived from secondary tests of samples that have been strained without bending, for comparison;

5. points derived from secondary tests as described in section 7.1;

6. points derived from samples showing failure by instability as described in section 7.2;

7. as 6 , but derived from tests with only two rolls instead of three, and applying a constant force (not for all materials).

8. points derived from interrupted tests as described in section 7.3 (only for materials MS2 and AL2).

It is easily noticed that the points derived from secondary tensile tests (types 3,4 and 5) coincide with the extrapolated curve, although with a fair amount of scatter. This is an extraordinary result because it suggests that the level of hardening is not at all affected by the cyclic bending operations contrary to what might be expected. This needs further investigation.

First of all it should be noticed that the points are derived from a wide variety of samples taken from all test series. This means that the forming history is complex, showing both variations in the number of bending cycles, and in the width of the cyclic zones II (Fig. 3). Yet, within an acceptable amount of scatter the results from all samples produce identical results. This is best illustrated by two grey points of type 5 marked A that almost coincide. One has been tested at $3.5 \mathrm{~mm} / \mathrm{s}$, the other at $0.35 \mathrm{~mm} / \mathrm{s}$. But both fail at the same elongation, meaning that one has been subjected to ten times as many bending cycles as the other, yet both show the same strength! This could imply that some situation of saturation is obtained after a few bending cycles. However, the points between strains of 0.2 and 0.4 can be compared directly to points derived from samples without any bending, and no difference is observed. This indeed strongly supports the conclusion that the overall strength of the 
samples after testing is not affected by the bending cycles, but only by the total length strain, and obeys the normal hardening relation.

The points taken from failure by instability (items type 6) show somewhat more scatter, and are slightly weaker, but otherwise present the same result. The reduced strength may well be caused by the inaccuracy in the measurements of the cross-section area, that yield a slightly too large value due to non-flatness of the specimens and roughening.

It is well known that low-C steel in general hardens by a power-law relation for low strains in good approximation, but it is also known that this is not always the case for high strains, notably above 1.0. Therefore it is not sure if the extrapolated curve indeed shows the correct hardening. A way to validate the curve would be for example to use samples taken from material rolled to various levels for comparison, but that has not been done here.

A compilation of the other mild steel results is presented in Fig. 17. Basically all materials show the same results, although materials MS3 and MS4 show considerably less scatter than other materials. These two materials have not been subjected to a complete series of tests comprising all combinations of settings, but to a restricted series of tests intended to be used as a reference. Therefore the majority of tests have been carried out at a setting that enabled the largest overall elongation, and the tests have been stopped at certain elongations to provide variations in strain. This shows that the level of scatter can be reduced significantly if the settings are chosen carefully, and also indicates that the inaccuracy in strain measurements is acceptable. The results of these tests have been used successfully as a reference to study material hardening in Incremental Sheet Forming [11]. Materials MS4 and MS5 (and other) show both type 6 and type 7 results, but within the scatter there is no difference between the two types. This further indicates that the strength is little affected by major experimental conditions. The same holds for results type 8 in material MS2 (and AL2). On the other hand, the fact that materials MS3 and MS4 show very low scatter suggests that the larger amount of scatter present in the other materials are not just measuring errors but reflect differences in mechanical properties, that however could not be simply related to differences in the test conditions. It is also observed that data taken from samples pulled to fracture in the CBT test seem to show more scatter than data taken from samples not pulled to fracture.

A compilation of the other two low-C steel results is presented in Fig. 18. Material MS6 is material MS1 but pre-strained, and the extrapolated curve for this material is just the extrapolated curve of material MS1 but shifted accordingly. Basically both materials show the same results as the other materials. Only for dual-phase steel the results type 6 seem to show a more systematic difference as compared to results type 5 at high strain. Dual phase steel also
Fig. 17 Constructed hardening curves for other mild steel variants. Legend as in Fig. 16
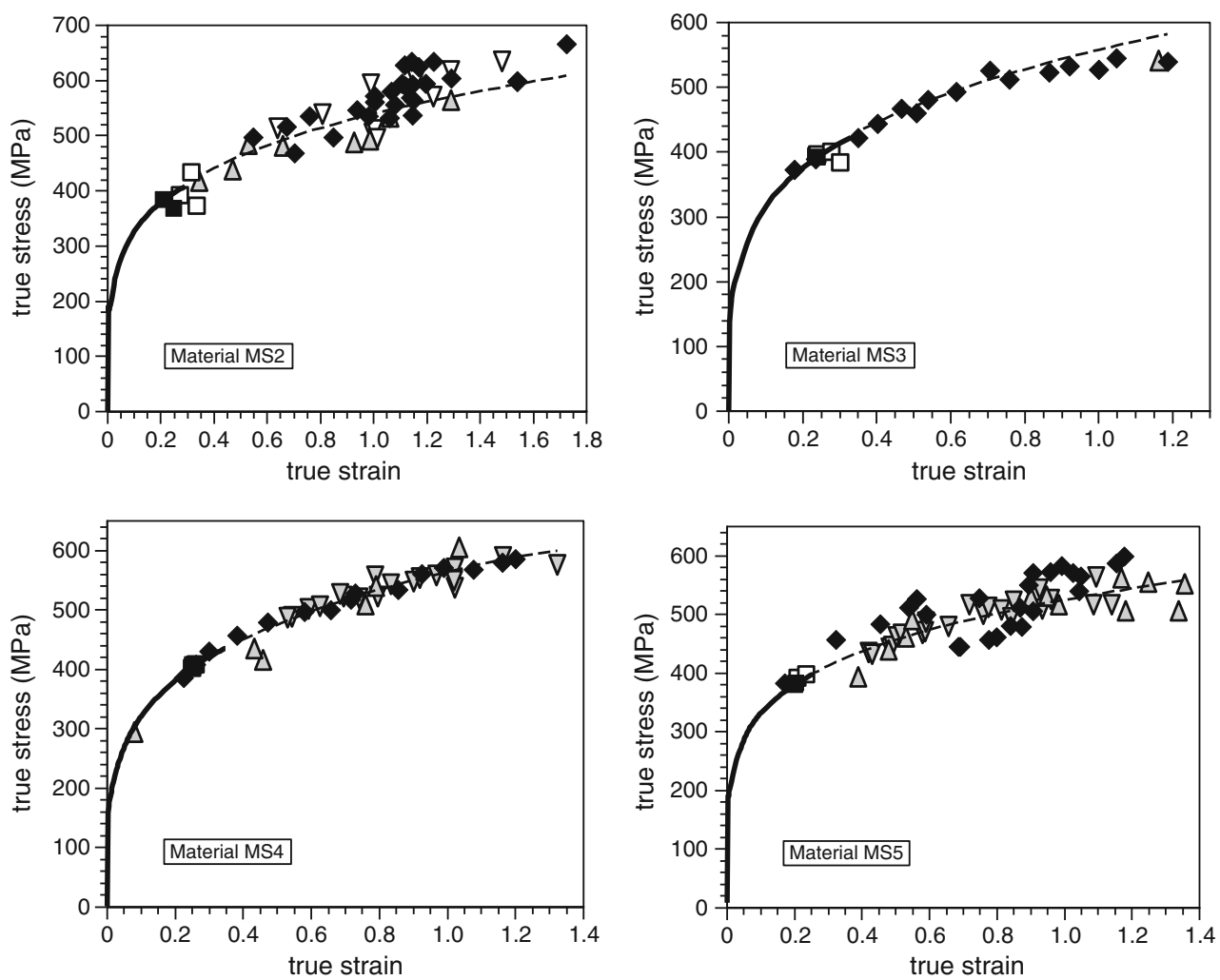
Fig. 18 Constructed hardening curves for other low-C steel variants. Legend as in Fig. 16

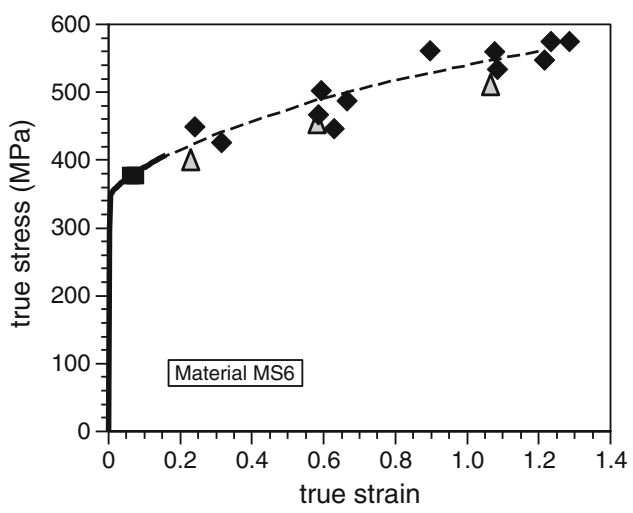

shows a secondary influence of experimental setting that will be discussed below.

A compilation of the results for the three aluminium variants is presented in Fig. 19, no extrapolated hardening curve is presented. The hardening curve differs from that for mild steel as expected. The small irregularities in the measured curve of material AL1 (AA 5182) in the strain range of $0.05-0.2$ are caused by serrated yielding. The aluminium results show less type 6 or type 7 results due to the particular fracture behaviour of that material. This figure also shows the curve for titanium. In general the results agree with those found for the low-C steel variants.

Finally, Fig. 20 presents the result for the three brass variants and stainless steel. So far the constructed hardening curve for high strains seemed simply a continuation of the measured curve for low strains. However brass seems to be an exception, and this is most noticeable for soft brass (BR1). Although for that material the results obtained after CBT testing do fit with the measured hardening curve at around 0.5 true strain, the rate of hardening (the slope) seems lower than that of a simple continuation of the measured curve. This is but a visual interpretation as reference data are missing, but it is noticeable. The effect is particularly noticeable when the data are compared to that for stainless steel. Both materials show almost identical hardening in a conventional tensile test (except for the level), yet the measured hardening after the CBT test differs clearly. It is known that yellow brass deforms by twinning, and a
Fig. 19 Constructed hardening curves for aluminium and titanium. Legend as in Fig. 16
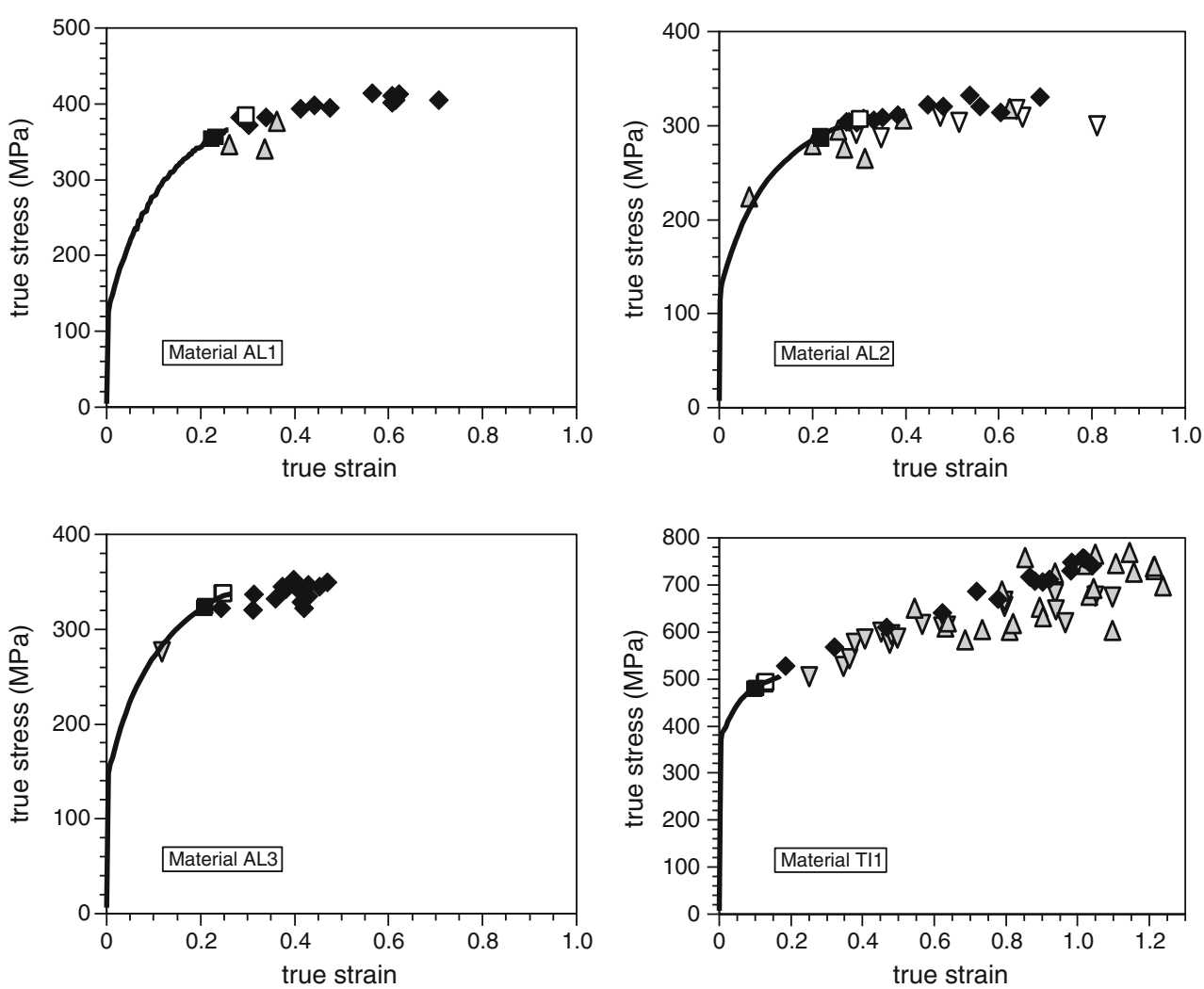
Fig. 20 Constructed hardening curves for brass and stainless steel. Legend as in Fig. 16
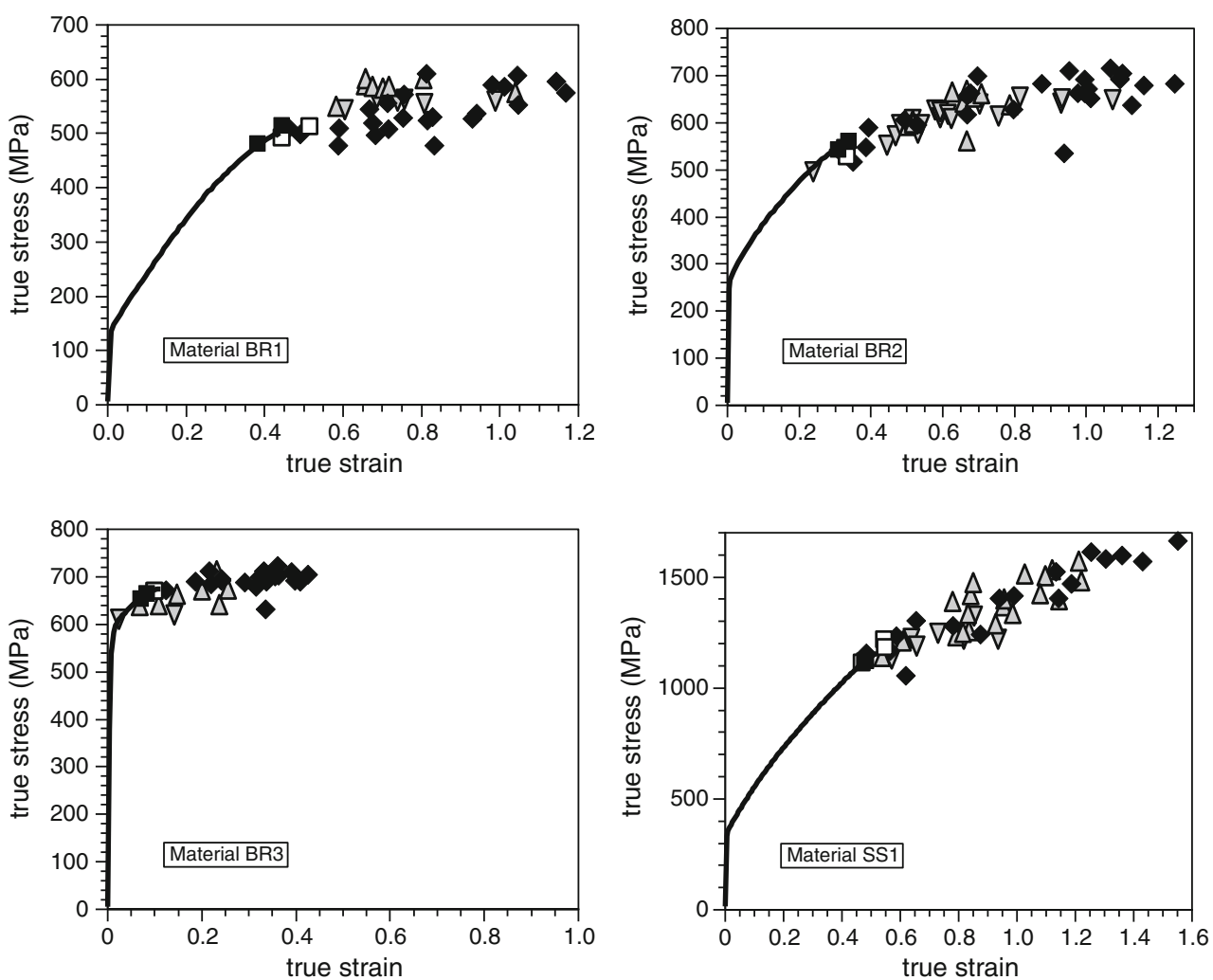

possible explanation could be that under the cyclic conditions encountered in the CBT test less twinning occurs. This is however purely speculative, and it is not clear how twinning affects hardening. Note further that material BR1 is the only material where the results of type 6 and 7 are on average actually higher than the points of type 5 , but this may be accidental.

Both multi phase materials (dual-phase steel and strained stainless steel) show an influence of experimental conditions that is not encountered in any of the other materials. This is presented in Fig. 21 that shows that a larger roll penetration (parameter P in Fig. 1) results in lower strength after the CBT test. Although the results are somewhat obscured by scatter, the tendency is clear. In tests with a higher penetration the bending radius is smaller, and consequently the bending strains are larger. This means that also the strain cycles as indicated in Fig. 3 by line 4 are larger. This considerably increases the total incremental strain, and this could be observed easily as a considerable heating of the specimens by the dissipation of mechanical work in tests with a higher roll penetration. This can explain the effect for stainless steel. SS304 is an austenitic stainless steel that partially transforms to martensite by plastic deformation, but the rate of transformation strongly depends on the temperature. So the higher temperature occurring in tests with higher depth setting causes less transformation and consequently a lower strength. This also indicates that this effect might be eliminated by performing tests at true constant temperature.
Fig. 21 Effect of roll penetration on final strength for stainless steel (left) and dual-phase steel (right). Only results type 5 and type 6 are shown
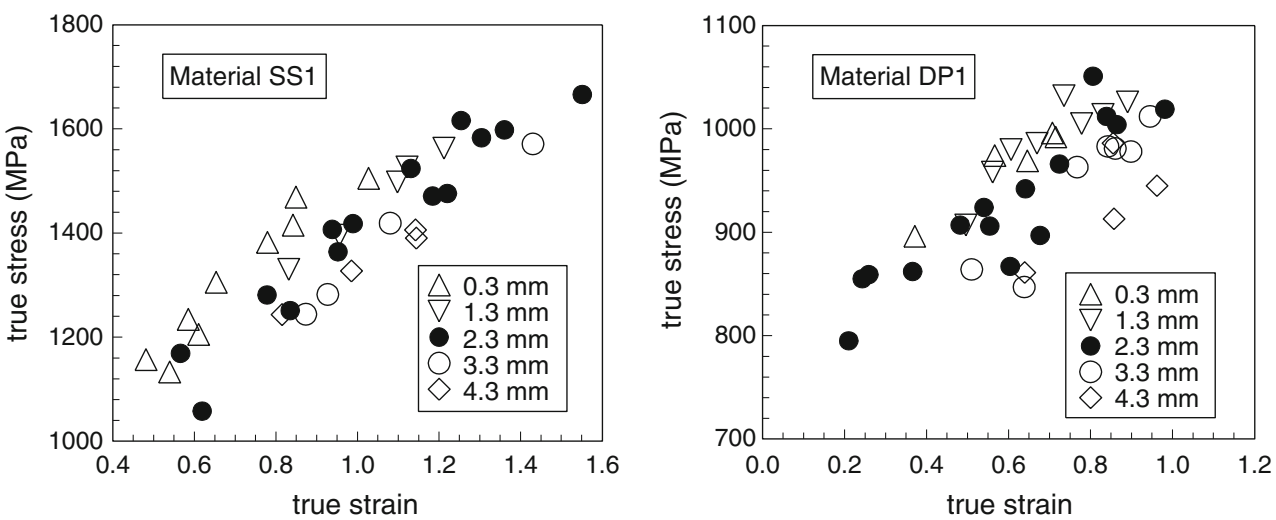
Similar effects are observed with dual-phase steel. The reason for this is unknown as it is unlikely that this is caused by a mechanism as described for stainless steel.

\section{Discussion of Results}

The results presented above lead to the following conclusions with only a few exceptions:

- the strength of the material after CBT testing is independent of the specific conditions during the CBT test

- the strength is only determined by the macroscopic length strain, and seems to follow the normal hardening relation of the material.

Note that the second conclusion automatically implies the first. This is a relevant observation as it implies that indeed the CBT test can be used as a means to characterize material at high strain levels, at least potentially. The underlying observation is that the cyclic strain history as shown in Fig. 3 does not affect final hardening. To be precise: it suggests that material that is subjected to cyclic straining recovers its 'original' strength when subjected to consecutive monotonous straining. Unfortunately there is little literature that either confirms or invalidates this assumption. Only few references have been found, and for low strains only. For example, steel sheet material can be flattened by so-called tension levelling that uses cyclic bending very similar to the CBT test; yet it is well known that this process barely affects the mechanical properties including the yield stress, despite the fact that the incremental cyclic strain may be as high as $10 \%$. Fatigue type experiments are also carried out frequently, but in general the strain level is very low. Kang and co-workers have carried out several ratcheting experiments and Fig. 22 presents a reprocessing of results published by Kang et al. [12]. The solid line presents the stress history in an experiment, where however

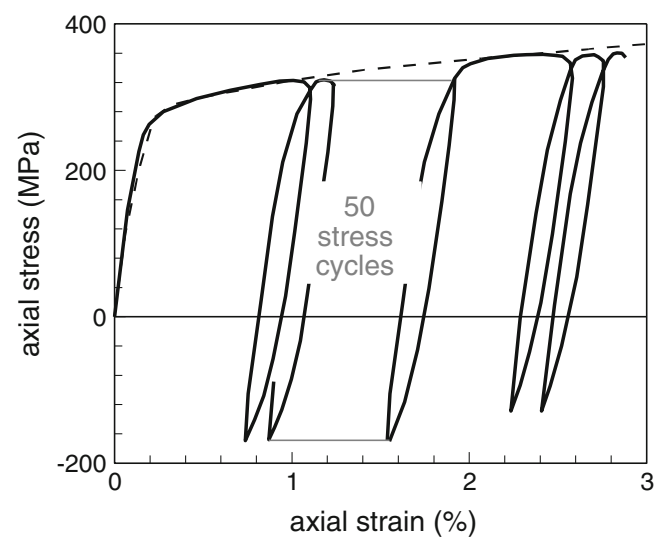

Fig. 22 Stress history in cyclic tests (solid line) compared to monotonous test (dashed line). Data from Kang et al. (2002) not all individual stress cycles are shown. The dashed line presents stress in a monotonous test. The results show that when the test is continued as a monotonous test after performing 50 stress cycles, the stress returns to the monotonous level. But as the strain levels in this and similar tests are much lower than in the CBT tests described in the present paper, this can only serve as an indication.

Cyclic straining to high strain levels can be carried out by torsion of thin-walled tubes. For example Meyer et al. have carried out an extensive test program on both aluminium and alloyed steel under a wide range of conditions, showing how the stress amplitude depends on experimental conditions [13]. The strain history in their tests is similar to that shown in Fig. 3, lines 3 and 4. But their cyclic tests have never been continued as a simple monotonous test, so there is no reference.

The fact that all materials present similar results (excluding some second order effects) strongly supports the conclusion that apparently materials recover their original strength after cyclic testing, when subjected to monotonous straining, but a thorough explanation has yet to be given. Brass seems to be an exception. It is known that brass deforms by twinning, but it is not known how this affects hardening in the CBT test. Recent work of Sakharova et al. showed that strain path changes promote the onset of premature twinning in brass [14], and as in the CBT test obviously the strain path frequently changes, this can affect hardening. But as the Sakharova tests are limited to strains of 0.3 , a direct translation of their results is not possible.

\section{Proposal}

A simple outlook is now that these tests can be used to construct hardening curves up to high levels of strain,

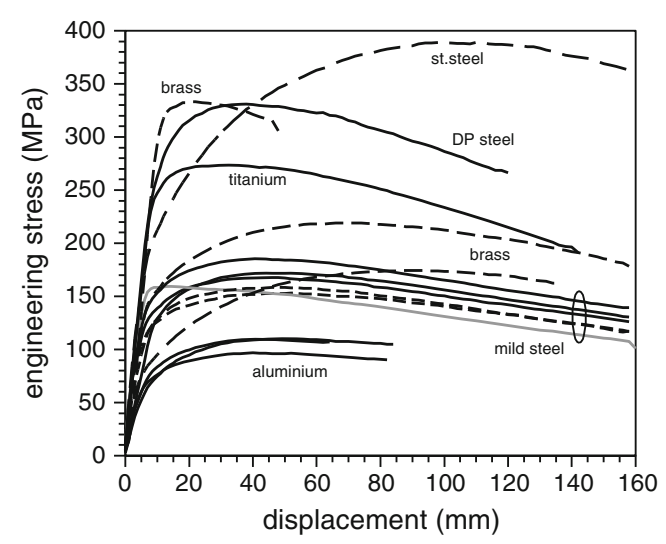

Fig. 23 Engineering stress-displacement curves for all materials, determined under the same experimental conditions. The process of smoothing may have affected the very first and last part of the curves 
certainly if some precautions are taken. The question arises if we need all that amount of work to construct a hardening curve, or if it can be obtained from a single recorded forcedisplacement curve. After all, at any moment the pulling force is a certain fraction of the true flow force defined as flow stress $\times$ cross-section area, see [7]. So it should be possible to obtain the flow stress from a single curve. The problem is that this fraction not only depends on the experimental conditions, but also changes during a single test, so a simple conversion is not possible. To further substantiate this proposal it can be checked if the individual measured force-displacement curves do exhibit typical material characteristics. This is done in Fig. 23 that presents a collection of force-displacement curves (after smoothing) for all materials, all obtained using the same experimental conditions. For easy comparison the force has been converted into engineering stress defined as the measured pulling force divided by the original cross-section area. It is clear that indeed these curves do show the characteristic hardening behaviour of the materials, most notably seen in the different elongations at maximum stress. The results also show an influence of thickness. All mild steel variants have very similar mechanical properties (Table 1), yet the dashed curves for the thicker variants MS2 and MS5 are clearly lower than the solid curves for the thinner variants, although the image is somewhat obscured by the grey curve for the pre-strained material MS6. To actually construct the hardening relation from such a single curve requires a much more thorough analysis of the test, but it should be possible.

\section{Final Discussion and Recommendations}

The aim of this study was to determine if the CBT test can be used to determine the strength of materials at high strain levels. Based on the consistent results for many different materials, the first, cautious conclusion is affirmative. The results presented here are taken from a test program that was aiming at formability, not material behaviour. Consequently the individual tests were not optimized for material characterization, with exception the test of materials MS3 and MS4 that show that the level of scatter can be reduced significantly if the settings are chosen carefully. The best results are obtained by performing secondary tensile tests under controlled conditions on samples taken from specimens that have been CBT tested but not until fracture. Nevertheless, confirmation from another laboratory is advisable, as is comparison to characterization of material elongated in another way like rolling. In this way the original tests proposed by Taraldsen [1] are ideal as they do not incorporate bending, but they are more difficult to perform than the CBT test. It might seem obvious to do an analysis, for example by FEM, but that would require a material model that is known to be able to cope with cyclic straining at high strains sufficiently accurate, and such models are still under development. The results presented here may help to develop or verify such advanced material models.

\section{Conclusions}

The strength of materials after cyclic stretch-bending has been measured for a wide variety of materials up to high levels of uniform elongation.

The strength is independent of the experimental details of the cyclic pre-deformation, with the exception of stainless steel and dual-phase steel that both show a slight influence of experimental conditions.

The level of hardening agrees with an extrapolation of the hardening in a conventional tensile test, notably for low-C steel where the hardening obeys a power law to high strain levels; only brass seems to be an exception.

The results indicate that potentially the CBT test can be used to determine the hardening relation of materials at high strain, but more research is required.

Open Access This article is distributed under the terms of the Creative Commons Attribution Noncommercial License which permits any noncommercial use, distribution, and reproduction in any medium, provided the original author(s) and source are credited.

\section{References}

1. Taraldsen A (1964) Stabilized tensile testing (i e without local necking). Materialprüfung 6(6):189-228

2. Rijken A (1965) De trekproef met gelijktijdig walsen. Hoogovens Internal Report 10165, March 1965 (in Dutch)

3. Benedyk JC, Stawarz D, Parikh NM (1971) A Method for Increasing Elongation Values for Ferrous and Nonferrous Sheet Metals. J of Materials 6(1):16-29

4. Taraldsen A (1972) Tension testing without local necking. J of Materials 7(4):470-473

5. Benedyk JC, Janota R (2002) The continuous bending under tension (CBT) test method for evaluating formability of sheet metals. Light Metal Age 2002:6-9

6. Emmens WC, van den Boogaard AH (2009) Incremental forming by continuous bending under tension-An experimental investigation. J Mat Proc Technology 209:5456-5463

7. Emmens WC, van den Boogaard AH (2011) Cyclic stretch-bending: mechanics, stability and formability. J Mat Proc Technology 211 (2011):1965-1981

8. Allwood JM, Shouler DR (2011) Design, analysis and application of a novel test for sheet metal forming limits under non-planar stress states. Proceedings 14th Int. Esaform. Conf. on Material Forming. AIP Conf Proc 1353:1595-1600

9. Emmens WC, van den Boogaard AH (2011) The stabilizing effect of bending-under-tension. Proceedings 14th Int Esaform Conf on Material Forming. AIP Conf Proc 1353:1583-1588 
10. Hadoush A, van den Boogaard AH, Emmens WC (2011) A numerical investigation of the continuous bending under tension test. J of Mat Proc Technology 211:1948-1956

11. Emmens WC, van den Boogaard AH (2009) Incremental sheet forming analysed by tensile tests. Proc. Shemet09. Birmingham, UK, 6-8 Aril 2009; Key. Eng Materials 410-411:347-354

12. Kang G, Gao Q, Cai L, Sun Y (2002) Experimental study on uniaxial and nonproportionally multiaxial ratcheting of SS304 stainless steel at room and high temperatures. Nucl Eng Des 216(2002):13-26

13. Meyer LW, Gahlert C, Hahn F (2005) Influence of an incremental deformation on material behaviour and forming limit of aluminium A199,5 and QT-Steel 42CrMo4. Proceedings 11th Shemet, Apr 0508, 2005, Erlangen, Germany, pp 417-424

14. Sakharova NA, Fernandes JV, Vieira MF (2009) Strain path and work hardening behavior of brass. Mat Science and Eng A 507:13-21 